$\begin{array}{ll}\text { Research Square } & \begin{array}{l}\text { Preprints are preliminary reports that have not undergone peer review. } \\ \text { They should not be considered conclusive, used to inform clinical practice, } \\ \text { or referenced by the media as validated information. }\end{array}\end{array}$

\title{
Analysis of the prognostic biomarkers associated with cancer stem cell characteristics and immune infiltration of Helicobacter pylori-induced gastric cancer
}

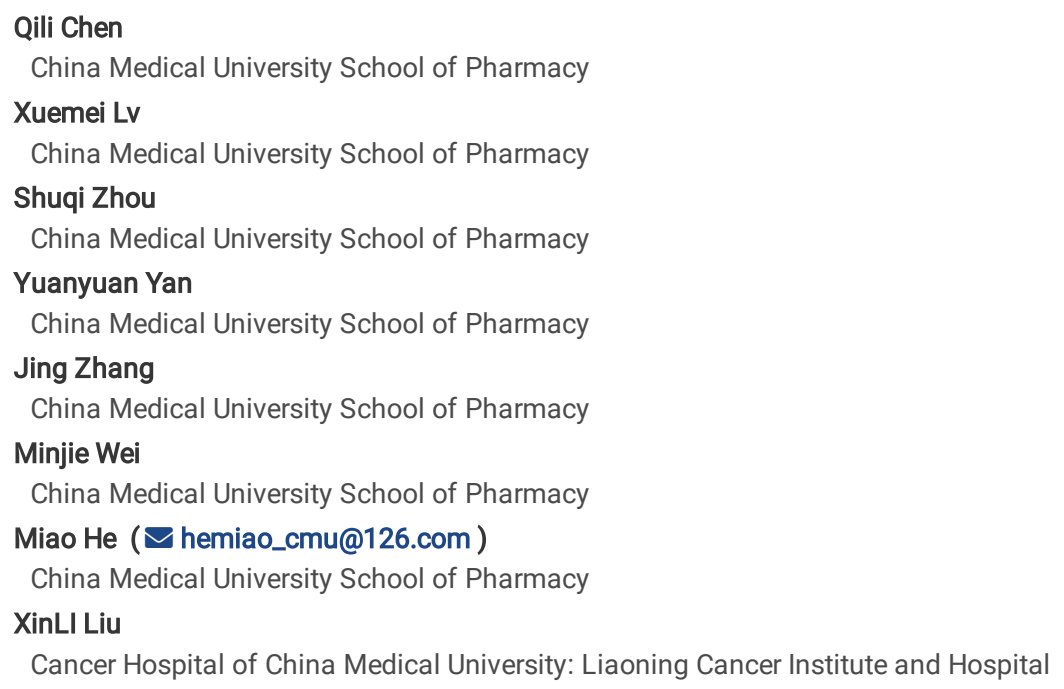




\section{Abstract}

Background

China is a country with a high incidence of gastric cancer, accounting for about half of the new cases in the world every year. The development of Gastric cancer (GC) is closely related to Helicobacter pylori (H. pylori) infection. Cancer stem cells (CSCs) play important roles in the survival, proliferation, metastasis and recurrence of tumors. However, the mechanism of CSCs and H. pylori-induced GC remains unclear.

At the same time, the effect of tumor microenvironment on tumor stem cells and GC with $\mathrm{H}$. pylori infection is also worth exploring. On the basis of the existing research on GC, it is still of great significance to further understand and explore the pathogenesis of GC through bioinformatics analysis.

\section{Results}

There was higher mRNA expression-based stemness index (mRNAsi) in H. pylori (+) GC tumor tissue than in normal tissue. The clinical features of stage T and $\mathrm{N}$ were highly correlated with mRNAsi. The purple module was the most significant modules related to the mRNAsi by WGCNA and its key genes played played a major function related to tumor immunity, mainly involved in regulating the Jak-STAT signal pathway, Th17 cell differentiation pathway, and so on. These key genes were correlated with the high level of immune infiltration of $\mathrm{CD} 8^{+} \mathrm{T}$ cells, CD $4^{+} \mathrm{T}$ cells, macrophages, neutrophils and dendritic cells in GC via Tumor Immune Estimation Resource (TIMER). 28 genes were found to be closely related to the characteristics of gastric CSCs (GCSCs) associated with H. pylori infection. 13 genes were associated with lymph node metastasis in GC, which may lead to poor prognosis in patients.

\section{Conclusion}

The characteristics of GCSCs were related to the interleukins signaling, cytokine signaling, Jak-STAT signaling pathway, which provides a new idea for screening therapeutic targets to inhibit the characteristics of GCSCs and a basis for the eradication of $\mathrm{H}$. pylori and the prevention of GC.

\section{Background}

Gastric cancer (GC) is one of the most common leading causes of cancer-related death around the world. According to global cancer statistics, there will be an estimated 27,600 new cases of invasive GC in the United States in 2020 [1]. The International Agency for Research on Cancer classified Helicobacter pylori (H. pylori) as the first category of human infectious carcinogen [2]. Many studies have shown that $\mathrm{H}$. pylori plays a significant role in GC [3-5]. Although many therapies have been improved recently, including surgery, chemotherapy, radiation therapy and targeted therapy, the overall 5-year survival rate of GC is still less than 30\% [6]. Tumor metastasis was one of the reasons for the poor prognosis of GC [7, 8]. In recent years, the theory of cancer stem cells (CSCs) has been recognized. CSCs, with the potential for self-renewal and unlimited spread, could cause postoperative recurrence and metastasis, and play a decisive role in the initiation of tumor formation and growth [9]. Some researchers have found that the stemness of metastatic tumor cells was higher, and there was a negative correlation between the stemness of tumor cells and survival rate. Tumor stemness may be the main cause of GC recurrence and drug resistance [10, 11]. At present, the important role of $\mathrm{H}$. pylori in the formation and development of GC stem cells (GCSCs) is still confusing.

Recently, an innovative one-class logistic regression machine-learning algorithm was used to provide novel stemness indices. MRNA expression-based stemness index (mRNAsi) was defined to reflect the gene expression features that can assess the degree of oncogenic dedifferentiation and evaluate the cancer progression [12]. Based on the identification and weighting of the associated expression levels between gene pairs, weighted gene co-expression Network Analysis (WGCNA) is constructed and widely used to process gene expression data and study network changes [13]. Using this logistic regression machine-learning algorithm, an increasing number of cancers has been analyzed, including breast cancer [14], liver hepatocellular carcinoma [15], lung adenocarcinoma [16], bladder cancer [17], and glioma [18] and so on, but there was no correlation analysis for GC. In order to evaluate the role of mRNAsi in GC, we adopted a method to identify genes related to stemness through WGCNA [19]. In addition, many studies have found that tumor cells were closely related to the surrounding tumor microenvironment (TME). The tumor immune infiltration present on tumor microenvironment is characterized by leukocyte infiltration, such as Tumor-associated macrophages and tumor-associated neutrophils, which can affect the prognosis of cancer patients and the effectiveness of chemical and immunotherapy $[20,21]$. Chronic inflammation of the stomach caused by $\mathrm{H}$. pylori infection plays main roles in the initiation and progression of GC [22].

In summary, our results provided reliable data support for further analysis of the potential relationship of H. pylori, GCSCs and immune infiltration, and showed stemness related genes (SRGs) could be a potential biomarker of GC. It may also provide novel insights about that SRGs affect inflammatory mediators (such as Cytokines and Chemokines) in H. pylori-induced GC. Meanwhile, it also provided a basis for clinical development of H. pylori eradication therapy and GC prevention.

\section{Results}

\section{The mRNAsi index is significantly correlated with $\mathrm{H}$. pylori-positive gastric cancer}

A total of $408 \mathrm{GC}$ samples and 31 normal samples were downloaded from TCGA database. We manually classified each GC sample into a patient with $\mathrm{H}$. pylori (-) or H. pylori (+) GC. Finally, after removing patients with incomplete clinical information, $142 \mathrm{H}$. pylori (-), $18 \mathrm{H}$. pylori (+) GC cases and 30 normal samples were obtained. The patients' mRNAsi index was from the appendix of the article[12]. Tumor tissues are composed of thousands of different cells, including tumor cells and other types of cells, such as stromal cells and immune cells[23]. In order to eliminate the mRNAsi bias caused by non-cancer cells in the tumors, we used ESTIMATE to calculate the tumor purity and correct the mRNAsi index as mRNAsi according to the previous report [14]. We found that the mRNAsi index of tumor group was higher than normal group $(P<0.0001$, Figure $1 \mathrm{~A})$, indicating the importance of mRNAsi in GC. Additionally, we compared the 
mRNAsi index of normal samples, H. pylori (-) GC samples and H. pylori (+) GC samples, and found that the index of H. pylori (+) GC samples was higher than that of $\mathrm{H}$. pylori (-) GC samples (Figure 1B), indicating that $\mathrm{H}$. pylori may be related to gastric CSCs. In the survival analysis, the patients with higher mRNAsi have shorter OS than those with lower mRNAsi though there was no statistical significance $(P=0.0861$, Figure $1 C)$.

\section{Screening of DEGs and key genes for mRNAsi}

In order to more specifically construct the gene co-expression network of GC, the data of $142 \mathrm{H}$. pylori (-), $18 \mathrm{H}$. pylori (+) GC cases and 30 normal samples mentioned above were filtered, standardized, and differentially analyzed. The DEGs between $\mathrm{H}$. pylori (+) and $\mathrm{H}$. pylori (-) GC samples were not got, but DEGs between H. pylori (+) GC samples and normal samples were obtained. Among them, a volcano map showed that 4,157 genes were up-regulated and 4,982 genes were down-regulated (Figure 2A). After cluster analysis, the DEGs with the highest variance were constructed a WGCNA co-expression network, and 13 modules were obtained for subsequent analysis (Figure 2B). Next, in order to analyze the relationship between each module and the mRNAsi of the sample, MS was used as the overall gene expression level of the corresponding module, and its correlation with mRNAsi was calculated (Figure 2C). The correlation between the purple module and mRNAsi is the most significant and R value of positive correlation is 0.79 . In addition, the blue module has a high negative correlation with mRNAsi, and the R value is -0.68 . The correlations between the purple or blue modules and the genes in these modules were 0.71 and 0.86 , respectively (Figure 2D-E). Finally, we selected the purple module for subsequent analysis to screen out key genes related to GC stemness. The selection criteria were defined as cor.MM $>0.8$ and GS>0.5. In the end, 28 key genes were screened out.

\section{Co-expression Network and Enrichment Analysis of Key Genes}

In order to explore the relationship between these key genes, we conducted a co-expression network analysis and found that the key genes in the purple module, such as interleukin receptor IL21R, tyrosine protein phosphatase non-receptor PTPN7, chemokine receptor CCR5, and tyrosine There is a strong correlation between the amino acid protein kinase JAK3, fibronectin receptor ITGA4, Schlafen family SLFN12L and other genes (Figure 3A) and statistically significant $(P<0.05)$. Then the STRING online database was used to perform a protein-protein interaction enrichment analysis on these key genes, and the relationship between the key genes and their protein products was determined, which indicates that these key genes form a very dense interaction network (Figure 3B). In order to clarify the functional similarity of the modular genes, a gene ontology (GO) term analysis was carried out on the genes in the modular. The main biological functions of the purple module were related to immune functions such as cellular response to cytokine stimulus, cytokine receptors and leukocyte migration (Figure 3C). Next, the process pathway enrichment analysis of KEGG and Reactome database was performed (Figure 3D). The results showed that these key genes were related to the interleukins signaling, cytokine signaling, Jak-STAT signaling pathway, Th17 cell differentiation pathway, chemokine signaling pathway and others. In addition, we selected the terms with similarity> 0.3 , and constructed a network graph through Metascape (Figure $3 \mathrm{E})$. The data indicate that these key genes and their pathways may be related to both mRNAsi and immune function. That is, the occurrence and development of GCSCs may be closely related to immunity.

\section{Analysis and Validation of Key Genes}

In order to further explore these key genes, we found the higher expression of these key genes in GC samples than normal tissues through GEPIA database analysis (Figure 4A). To systematically understand the expression levels of these key genes, we used Oncomine database to analyze their expression in other cancer types (Figure 4B). And we found that most of all key genes were obviously upregulated in more than one cancer type.

\section{Effect of Lymph Node Metastasis on the mRNAsi Key Genes and Clinical Prognosis in GC}

We next used Kaplan-Meier database to explore the relationship between the expression levels of key genes related to stemness and the prognosis of GC patients, and found high expression of 13 genes, including CCR5, EOMES, FGD3, GRAP, IL24, IL27RA, JAK3, P2RX5, PARP15, PCED1B, PTPN6, PTPN7 and TMC8, may lead to poor prognosis of patients (Figure 5). Lymph node metastasis is an important factor affecting the prognosis of GC patients. Thus, we used Kaplan-Meier plotter to determine the effect of lymph node metastasis on the key genes mRNA expression and clinical prognosis in GC. Here the N category refers to lymph node involvement; N1-N3 indicate regional lymph node metastasis. High mRNA expression of key genes is associated with poor OS in gastric cancer patients. Specifically, key genes expression has the high HR values of OS in the five $\mathrm{N}$ categories. As shown in Table 1, the expression levels of key genes were related to the lymph node metastasis (All, N1, N2, N3, N1+2+3) of GC patients, and most of genes expression have the high HR value (PTPN6, N1, $\mathrm{HR}=2.98, P<0.001$; CCR5, N1, HR $=2.06, P<0.001)$. OS may be affected by lymph node metastasis in $\mathrm{GC}$ patients.

Table.1 Kaplan-Meier plotter to determine the effect of lymph node metastasis on the key genes mRNA expression and clinical prognosis in GC 


\begin{tabular}{|c|c|c|c|c|c|c|c|c|c|c|c|c|c|c|c|}
\hline & ALL & & & N1 & & & N2 & & & N3 & & & $\mathrm{N} 1+2$ & & \\
\hline Genes & $\mathrm{HR}$ & $95 \% \mathrm{Cl}$ & $\mathrm{P}$ & $\mathrm{HR}$ & $95 \% \mathrm{Cl}$ & $\mathrm{P}$ & $\mathrm{HR}$ & $95 \% \mathrm{Cl}$ & $\mathrm{P}$ & $\mathrm{HR}$ & $95 \% \mathrm{Cl}$ & $\mathrm{P}$ & $\mathrm{HR}$ & $95 \% \mathrm{Cl}$ & $\mathrm{P}$ \\
\hline CCR5 & 1.24 & $1.04-1.48$ & * & 2.06 & $1.31-3.23$ & * & 1.66 & $1.03-2.7$ & * & 1.38 & $0.81-2.36$ & 0.23 & 1.58 & $1.2-2.09$ & $\star \star$ \\
\hline EOMES & 1.25 & $1.01-1.56$ & * & 1.72 & $1.14-2.59$ & * & 0.63 & $0.4-0.99$ & * & 1.38 & $0.71-2.67$ & 0.34 & 1.31 & $1-1.7$ & * \\
\hline FGD3 & 1.8 & $1.4-2.32$ & $\star \star \star *$ & 2.77 & $1.75-4.39$ & $\star \star \star$ & 1.64 & $1.03-2.61$ & * & 1.92 & $1.11-3.32$ & * & 1.9 & $1.44-2.51$ & $\star \star \star$ \\
\hline GRAP & 1.49 & $1.18-1.89$ & $\star \star \star *$ & 1.76 & $1.13-2.74$ & * & 0.68 & $0.41-1.11$ & 0.12 & 2.58 & $1.44-4.64$ & $\star \star$ & 1.65 & $1.24-2.19$ & $\star \star \star$ \\
\hline IL24 & 1.24 & $1.03-1.5$ & * & 0.68 & $0.44-1.02$ & 0.06 & 1.39 & $0.83-2.34$ & 0.21 & 1.36 & $0.78-2.36$ & 0.27 & 0.79 & $0.61-1.03$ & 0.08 \\
\hline IL27RA & 1.37 & $1.15-1.62$ & $\star \star \star *$ & 1.72 & $1.11-2.66$ & * & 0.69 & $0.42-1.13$ & 0.14 & 1.97 & $1.1-3.52$ & * & 1.51 & $1.13-2.01$ & * \\
\hline JAK3 & 1.39 & $1.11-1.72$ & $\star \star$ & 1.78 & $1.18-2.68$ & * & 0.6 & $0.38-0.95$ & * & 1.84 & $1.08-3.14$ & * & 1.38 & $1.06-1.8$ & * \\
\hline P2RX5 & 1.41 & $1.17-1.69$ & $\star \star *$ & 2.26 & $1.49-3.42$ & $\star \star *$ & 1.34 & $0.84-2.15$ & 0.22 & 1.55 & $0.86-2.82$ & 0.15 & 1.49 & $1.5-1.94$ & * \\
\hline PARP15 & 1.28 & $1.03-1.59$ & * & 1.85 & $1.23-2.79$ & * & 1.67 & $1.07-2.61$ & * & 0.68 & $0.37-1.23$ & 0.2 & 1.39 & $1.06-1.81$ & * \\
\hline PCED1B & 1.5 & $1.18-1.91$ & $\star \star \star *$ & 2.3 & $1.43-3.72$ & ** & 0.77 & $0.49-1.21$ & 0.26 & 1.37 & $0.74-2.51$ & 0.31 & 1.64 & $1.22-2.2$ & ** \\
\hline PTPN6 & 2.11 & $1.74-2.56$ & $\star * *$ & 2.98 & $1.94-4.58$ & $\star \star \star \star$ & 1.93 & $1.22-3.04$ & * & 1.97 & $1.13-3.42$ & * & 1.92 & $1.47-2.49$ & $\star \star \star ~$ \\
\hline PTPN7 & 1.64 & $1.36-1.99$ & $\star * *$ & 2.33 & $1.52-3.57$ & $\star \star \star \star$ & 1.55 & $0.98-2.45$ & 0.06 & 1.74 & 0.89-3.4 & 0.1 & 1.71 & $1.3-2.26$ & 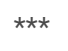 \\
\hline TMC8 & 1.34 & $1.07-1.68$ & * & 1.6 & $1.05-2.43$ & * & 0.79 & $0.49-1.29$ & 0.34 & 1.74 & $1-3.02$ & * & 1.36 & $1.04-1.78$ & * \\
\hline
\end{tabular}

*P $<0.01 ; * * \mathrm{P}<0.001 ; * * * \mathrm{P}<0.0001$

\section{Relationship between mRNAsi Key Genes Expression and Immune Infiltration}

To evaluate the most significant relationship between stemness and immune infiltration, we used MM>0.9 and GS $>0.5$ to select 5 genes, CCR5, JAK3, P2RX5, PARP15 and TIMC8, that affect the prognosis of GC patients for the next analysis. TIMER was used to calculate the abundance of 6 TIIC subpopulations (B cells, $C D 8^{+} T, C D 4^{+} T$ cells, Macrophages, Neutrophils and Dendritic Cells), and predicted the correlation between 5 key genes and immune cell infiltration level (Figure 6). B cells, CD8 ${ }^{+} \mathrm{T}$ cells, and NK cells were important anti-cancer effector cells in cancer. The results showed that, except for the low negative correlation between CCR5 and B cells, the expression of other key genes was positively correlated with the immune characteristics of GC. The results indicate that these key genes were closely related to the immune infiltration process of $\mathrm{GC}$, especially the regulation of $\mathrm{CD} 8^{+} \mathrm{T}$ cells, CD $4^{+} \mathrm{T}$ cells and Dendritic Cell infiltration.

We further used the GEPIA database to evaluate the correlation between the expression of key genes and immune markers, such as Monocyte markers (CD86, CD115), TAM markers (CCL2, IL10), M1 Macrophages markers (INOS, IRF5, COX2) and M2 Macrophage markers (CD163, VSIG4, MS4A4A) in GC Cells. In particular, SRGs were significantly correlated with M1 macrophage markers (INOS, IRF5, COX2) and M2 macrophage markers (CD163, VSIG4, MS4A4A) in GC. There is a relatively weak correlation between SRGs and the expression of M1 macrophage gene markers, while there is a relatively strong correlation between M2 macrophage markers CD163, VSIG4 and MS4A4A (Table 2). This suggests that in GC, SRGs may be capable of regulating the polarization of macrophages. 
Table 2

Correlation analysis between mRNAsi key genes and markers of monocyte, TAM and macrophages in GEPIA

\begin{tabular}{|c|c|c|c|c|c|c|c|c|c|c|c|c|c|c|c|c|c|c|}
\hline \multirow[t]{3}{*}{ Description } & \multirow[t]{3}{*}{ markers } & \multicolumn{4}{|c|}{ PARP15 } & \multicolumn{4}{|c|}{ CCR5 } & \multicolumn{4}{|l|}{ JAK3 } & \multicolumn{4}{|c|}{ P2RX5 } & \multirow{2}{*}{$\frac{\mathrm{TN}}{\mathrm{T}}$} \\
\hline & & $T$ & & $\mathbf{N}$ & & $T$ & & $\mathbf{N}$ & & $\mathrm{T}$ & & $\mathbf{N}$ & & $\mathrm{T}$ & & $\mathbf{N}$ & & \\
\hline & & $\mathrm{R}$ & $P$ & $\mathrm{R}$ & $P$ & $\mathrm{R}$ & $P$ & $\mathrm{R}$ & $P$ & $\mathrm{R}$ & $P$ & $\mathrm{R}$ & $P$ & $\mathrm{R}$ & $P$ & $\mathrm{R}$ & $P$ & $\mathrm{R}$ \\
\hline \multirow[t]{3}{*}{ M } & CD86 & 0.45 & $\star \star \star *$ & 0.5 & $\star \star \star *$ & 0.8 & $\star \star \star$ & 0.75 & $\star \star \star$ & 0.55 & $\star \star \star$ & 0.8 & 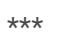 & 0.3 & $\star \star \star ~$ & 0.81 & $\star \star \star$ & 0.4 \\
\hline & CD115 & 0.42 & 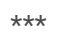 & 0.57 & $\star \star \star *$ & 0.76 & $\star \star \star ~$ & 0.63 & $\star * *$ & 0.54 & $\star \star \star$ & 0.65 & $\star * *$ & 0.22 & $\star \star \star ~$ & 0.62 & $\star \star \star *$ & 0.4 \\
\hline & (CSF1R) & & & & & & & & & & & & & & & & & \\
\hline \multirow[t]{3}{*}{ TAM } & CCL2 & 0.26 & $\star \star \star$ & 0.04 & 0.82 & 0.47 & $\star \star \star ~$ & -0.27 & 0.11 & 0.42 & $\star \star \star$ & -0.07 & 0.7 & 0.14 & $\star \star \star ~$ & -0.06 & 0.72 & 0.2 \\
\hline & CD68 & 0.23 & $\star * *$ & 0.12 & 0.47 & 0.5 & $\star \star \star *$ & 0.45 & $\star \star * *$ & 0.26 & $\star \star \star ~$ & 0.41 & * & 0.11 & * & 0.43 & $\star * *$ & $0 . \approx$ \\
\hline & IL10 & 0.37 & $\star * \star$ & 0.51 & $\star \star * *$ & 0.62 & $\star \star \star ~$ & 0.49 & $\star \star \star *$ & 0.47 & $\star \star * *$ & 0.6 & $\star * *$ & 0.19 & $\star * *$ & 0.6 & $\star \star *$ & $0 . \varepsilon$ \\
\hline \multirow[t]{5}{*}{ M1 } & INOS & 0.1 & * & 0.13 & 0.45 & 0.16 & $\star \star$ & 0.15 & 0.39 & 0.14 & $\star \star \star ~$ & 0.18 & 0.29 & 0.1 & * & 0.2 & 0.25 & 0.1 \\
\hline & (NOS2) & & & & & & & & & & & & & & & & & \\
\hline & IRF5 & 0.32 & $\star \star \star *$ & 0.3 & 0.07 & 0.38 & $\star \star \star *$ & 0.14 & 0.43 & 0.38 & $\star \star \star$ & 0.37 & * & 0.26 & $\star \star \star$ & 0.19 & 0.26 & 0.4 \\
\hline & cox2 & 0.09 & 0.06 & 0.12 & 0.49 & 0.04 & 0.37 & -0.24 & 0.16 & 0.07 & 0.16 & -0.11 & 0.52 & 0.02 & 0.69 & -0.14 & 0.43 & -0. \\
\hline & (PTGS2) & & & & & & & & & & & & & & & & & \\
\hline \multirow[t]{3}{*}{ M2 } & CD163 & 0.37 & $\star \star * *$ & 0.12 & 0.5 & 0.65 & $\star \star \star *$ & -0.13 & 0.44 & 0.47 & $\star * \star$ & -0.02 & 0.93 & 0.18 & $\star \star \star *$ & -0.04 & 0.84 & 0.4 \\
\hline & VSIG4 & 0.28 & $\star * *$ & 0.14 & 0.4 & 0.62 & $\star \star \star$ & 0.22 & 0.2 & 0.38 & $\star * \star$ & 0.18 & 0.3 & 0.11 & * & 0.29 & 0.09 & $0 . \approx$ \\
\hline & MS4A4A & 0.44 & $\star \star \star *$ & 0.29 & 0.08 & 0.73 & $\star \star \star *$ & 0.11 & 0.52 & 0.47 & $\star \star \star *$ & 0.18 & 0.28 & 0.23 & $\star \star \star *$ & 0.24 & 0.16 & 0.4 \\
\hline
\end{tabular}

Tumor, correlation analysis in tumor tissue of TCGA. Normal, correlation analysis in normal tissue of TCGA

*P $<0.01 ; * \star P<0.001 ; * \star * P<0.0001$

\section{Discussion}

Cancer is increasingly becoming a global health problem [24]. GC is the third-leading cause of cancer-related deaths worldwide, especially H. pylori is considered as an important risk of developing GC $[25,26]$. Despite the rapid development of GC genomics and proteomics has accelerated the identification of biomarkers, promoted prognosis prediction and personalized treatment, GC metastasis is one of the main reasons for treatment failure, which severely reduces the survival rate of patients, making GC still a problem that plagues surgeons and oncologists [27, 28]. It is believed that CSCs are the source of cells for the immortal proliferation and recurrence of malignant tumors [29]. CSCs are involved in tumor cells proliferation, invasion, metastasis and treatment resistance, leading to postoperative recurrence and metastasis, and ultimately predicting a low survival rate [30]. GCSCs are closely involved in occurrence and development of GC [31]. Therefore, it is extremely important to study the key genes that can drive the transformation of GCSCs.

In this study, we analyzed the relationship between mRNAsi and clinical characteristics from H. pylori (+) (-) GC samples and normal samples, and found that tumor tissues had higher stemness than normal tissues, and the corrected mRNAsi index was increased with the severity of tumors. More interestingly, we also found that $\mathrm{H}$. pylori (+) tumor tissue stemness $>\mathrm{H}$. pylori (-) tumor tissue stemness>normal tissue stemness. This important finding of the significance of $\mathrm{H}$. pylori on the GCSCs development is consistent with previous reports [32-34], which suggest that identifying key biomarkers associated with CSCs characteristics of $\mathrm{H}$. pylori-induced GC is very necessary.

We used WGCNA, a new method, to construct a gene network for the DEGs between H. pylori (+) GC tissues and normal tissues. Finally, 28 SRGs closely related to mRNAsi were obtained. These genes were not only up-regulated in the H. pylori (+) GC tissues, most genes were up-regulated throughout GC and other cancers. Gene function annotation and signal pathway analysis showed that these genes formed a complex network with strong interactions. The key genes that were highly related to mRNAsi mainly play roles in tumor immunity. They may also become new therapeutic targets for H. pylori (+) GC therapy. SRGs included members of the interleukin receptor family: IL21R, IL24, IL27RA, and IL2RB. IL-21R, as a carcinogen, was involved in the immune regulation of immune cells and the progression of a variety of malignant tumors [35-37], and also promoted the growth, migration and invasion of GC [38]. IL2RB polymorphisms were associated with lung cancer risk in the Chinese Han population [39, 40].

CCR5, a member of the CC chemokine family, was correlated with inflammatory diseases and cancers. In addition, the expression of CCR5 has also been shown to be an independent indicator of poor prognosis of GC [41]. JAK3 involved a series of physiological and cellular processes, such as cell proliferation, stem cell self-renewal and immune response. JAK3 mutations have also been described as contributing to GC [42]. It was also involved in the angiogenesis and metastasis of non-small-cell lung cancer [43]. PTPN7, an important member of T cell signal transduction, controls extracellular signal-regulated protein kinase 1/2 (ERK1/2) and p38 mitogen-activated protein kinase (MAPK) in T lymphocytes [44]. These genes play important roles in regulating the Jak-STAT signal pathway, Th17 cell differentiation pathway, chemokine signal pathway, interleukin signal pathway, T lymphocytes and B lymphocytes, which are related to both mRNAsi and immune function. 
H. pylori can cause inflammation [45], recruit more immune cells, and induce the release of a large number of inflammatory factors. Inflammatory factors can change the microenvironment of gastric pluripotent stem cells, promote epithelial-mesenchymal transition [46], and lead to the production, invasion and metastasis of GCSCs. Meanwhile, there are some other genes that have not been or rarely reported, such as MCOLN2, SNX22, SLFN12L, ST8SIA4 and so on. SRGs may be potential biomarkers and therapeutic targets for GC. Study on these SRGs may provide a clinical treatment idea for GC therapy targeting CSCs, such as CCR inhibitors and Jak inhibitors.

Another important aspect of our analysis is the first time to use mRNAsi to assess the correlation between the expression of SRGs and the immune infiltration and various levels of immune infiltration in GC. In different microenvironments, macrophages can be divided into classical activation (M1 phenotype) and alternative activation (M2 phenotype). M2 phenotype has immunomodulatory effects, such as participating in inducing Th2 response, promoting tumor angiogenesis and accelerating local lymph node metastasis $[47,48]$. Our results indicated that in GC, the expression levels of SRGs were correlated with the high level of immune infiltration of $\mathrm{CD}^{+} \mathrm{T}$ cells, $\mathrm{CD} 4^{+} \mathrm{T}$ cells, macrophages, neutrophils and dendritic cells in GC. In addition, the correlation between SRGs and M1, M2 markers also revealed that SRGs can potentially regulate TAM polarization, recruit immune infiltrating cells and regulate GC tumor immunity.

\section{Conclusions}

In summary, our study includes the correlation between immune infiltration and SRGs, which highlight the impact of immune infiltration on tumors. It is speculated that $\mathrm{H}$. pylori may affect the immune microenvironment and promote the progression of GCSCs. Our results provided reliable data support for further analysis of the potential relationship of H. pylori, GCSCs and immune infiltration. It will provide new ideas for studying the occurrence, development and treatment of GC. Meanwhile, it will also provide a basis for clinical development of H. pylori eradication therapy and GC prevention.

\section{Methods \\ Data collection and processing}

In this study, a total of 408 GC samples and 31 normal samples were enrolled. The Level 3 RNA-seq data (HTSEQ-FPKM-UQ)of patients with GC and clinical datasets containing information on $\mathrm{H}$. pylori were downloaded from TCGA of the National Cancer Institute (TCGA, https://cancergenome.nih.gov/). Then, we manually classified each GC sample into a patient with $\mathrm{H}$. pylori (-) or H. pylori (+) GC. After removing patients with incomplete clinical information, we got 20 samples with $\mathrm{H}$. pylori (+) and 168 samples with $\mathrm{H}$. pylori (-). The mRNAsi index that used to match with TCGA GC datasets was obtained from attachment of previous studies by Tathiane M. Malta [12]. The mRNAsi index and TCGA data of GC were merged. Finally, we got 18 tumor samples with H. pylori (+), 142 tumor samples with $\mathrm{H}$. pylori (-) and 30 normal samples. Tumor purity was calculated by CIBERSORT [49].

\section{Clinical Characteristic Correlation Analysis}

We used the Wilcoxon Signed Rank test to determine the corrected mRNAsi differences between tumor and normal tissues. Kruskal-Wallis test was used to determine the significant differences in tumor subtypes, stages, and TNM stages. Analyze the overall survival of corrected high-mRNAsi group and corrected low-mRNAsi group. GraphPad Prism version 8 (64 bit) was used to perform the above analysis. $\mathrm{P}<0.05$ was considered a significant difference.

\section{Screening of Differentially Expressed Genes (DEGs)}

We chose log2(TPM+1) to transform the expression data. Differentially expressed analysis was performed using the package "limma" [50]. The inclusion criteria for DEGs were log2-fold change $(\log 2 \mathrm{FC})>1$ and adjusted $\mathrm{p}$ (false discovery rate, FDR) $<0.05$. The volcano plot was drawn by R software.

\section{WGCNA analysis}

\section{Gene Co-expression Network Establishment}

The WGCNA R package was used to perform the subsequent analyses [19]. We selected the DEGs to ensure heterogeneity and accuracy of bioinformatics. In order to construct a gene co-expression network more targeted, firstly filter, standardize and analyze the difference of the sample data. Subsequently, the coexpression network conforms to the scale-free network, the logarithm $\log (\mathrm{k})$ of the occurrence of a node with a connection degree of $\mathrm{k}$ is negatively correlated with the logarithm $\log (\mathrm{P}(\mathrm{k}))$ of the occurrence probability of the node, and the correlation coefficient is greater than 0.8 . In order to ensure that the network is a scale-free network, we choose a soft threshold of $\beta=6$. The expression matrix is converted into an adjacency matrix, and then the adjacency matrix is converted into a topological matrix. Based on TOM, we use the average-linkage hierarchical clustering method to cluster genes, according to the standard of hybrid dynamic shearing tree, and set each gene. The minimum number of network modules is 30 . After determining the gene modules using the dynamic shearing method, we calculate the eigengenes of each module in turn, and then perform cluster analysis on the modules, and merge the modules that were closer to each other into a new module.

\section{Identifying Significant Modules and Key Genes}

In order to find modules that were highly relevant to mRNAsi, we chose mRNAsi and EREG-mRNAsi as the sample traits to find CSCs-related modules and genes. According to the expression level of each gene in each sample, we calculated the correlation between the genes in these modules and each sample traits to measure the degree of association between the gene and the sample traits (GS). The module eigengenes (MEs) function was used as a key part of the principal component analysis (PCA) for each gene module. In a certain module, the expression model of each gene can be summarized as an expression pattern with a distinct expression feature. Module significance (MS) was represented the correlation between the module and sample traits. A cutoff value $(<0.25)$ was used to screen and merge quite similar modules., and the modules that had the largest MS were considered the most sample trait-related modules. We selected the module with the strongest correlation through the MS value and calculated the GS and module membership (MM, correlation 
between gene expression profiles and module genes) for each gene. We defined the thresholds for the selection of key genes correlating with mRNAsi index in a certain module as $\mathrm{GS}>0.5$ and cor. gene $\mathrm{MM}>0.8$.

\title{
Co-expression Analysis and Relationships and Interactions analysis of Key Gene
}

The R corrplot package was utilized to analyze based on the gene expression levels the co-expression relationships between candidate genes by Pearson's correlation analysis. The correlation coefficient $>0.3$ and $p$-value $<0.01$ were considered statistically significant. We evaluated the protein-protein interaction (PPI) among key genes by STRING (version 11.0, https://string-db.org/), which is an online database that can be used to study and visualize the network of interactions among proteins.

\section{Functional Annotation and Pathway Enrichment Analysis}

The STRING [51] was used to perform functional enrichment for Gene Ontology (GO), Kyoto Encyclopedia of Genes and Genomes (KEGG) and Reactome analyses of the selected module. GO analysis consists of three terms: biological process (BP), cellular component (CC), and molecular function (MF). An adjusted $\mathrm{P}<0.05$ was used as the threshold. Comprehensive functional analysis of candidate genes was performed by Metascape (http://metascape.org/) [48], which clustered all enriched functional terms into nonredundant groups.

\section{Validation of Key Genes}

Oncomine (http://www.oncomine.org) [52] and GEPIA (http://gepia.cancer-pku.cn/) [53] were used to verify the mRNA expression of key genes between tumor and normal tissues in GC as well as other malignant tumors. The threshold of Oncomine screening was: p-value, 1E-4; fold change, 2; gene level, top 10\%.

\section{Expression and the prognosis of Key Genes}

The online database KM-plotter (http://www.kmplot.com/) was used to draw for overall survival in all GC patients between differential expression of key genes to examine the prognosis value and analyze the impact of clinicopathological factors on patient outcome.

\section{TIMER Database Analysis}

TIMER [54] is a comprehensive resource for systematic analysis of immune infiltrates across diverse cancer types (https://cistrome.shinyapps.io/timer/). TIMER applies a deconvolution previously published statistical method to infer the abundance of tumor-infiltrating immune cells (TIICs) from gene expression profiles, and estimate tumor immune infiltration by neutrophils, macrophages, dendritic cells, B cells and CD4/CD8 T cells.

\section{Gene Correlation Analysis in GEPIA}

We further assessed the link between key gene expression and the expression of particular markers associated with immune cell infiltration of tumors by using an online database GEPIA.

\author{
Abbreviations \\ GC \\ Gastric cancer \\ H. Pylori \\ Helicobacter pylori \\ cSCs \\ Cancer stem cells \\ TME \\ tumor microenvironment \\ WGCNA \\ Weighted gene coexpression network analysis \\ TCGA \\ The Cancer Genome Atlas \\ os \\ Overall survival \\ PPI \\ protein-protein interaction \\ GO \\ gene ontology \\ KEGG \\ Kyoto Encyclopedia of Genes and Genomes \\ MF \\ molecular functions \\ BP \\ biological processes \\ CC \\ cellular components \\ TIMER
}


Tumor Immune Estimation Resource

GCSCs

gastric CSCs

SRGs

stemness related gene

DEGs

Differentially Expressed Genes

GS

gene and the sample traits

$\mathrm{MM}$

module membership

\section{Declarations}

\section{Ethics approval and consent to participate}

Not applicable.

\section{Consent for publication}

Not applicable.

\section{Availability of data and materials}

All data analyzed in this study were prepared from databases including TCGA, GEPIA, KM-plotter and TIMER.

\section{Competing interests}

The authors declare that they have no competing interests.

\section{Author details}

1 School of Pharmacy, China Medical University, Shenyang, Liaoning Province, China

2 Liaoning Key Laboratory of molecular targeted anti-tumor drug development and evaluation; Liaoning Cancer immune peptide drug Engineering Technology Research Center; Key Laboratory of Precision Diagnosis and Treatment of Gastrointestinal Tumors, Ministry of Education; Shenyang, Liaoning Province, China

3 Shenyang Kangwei Medical Laboratory Analysis Co. LTD

4Department of Digestive Oncology, Cancer Hospital of China Medical University. Liaoning Cancer Hospital \& Institute. No.44 Xiaoheyan Road, Dadong District, Shenyang 110042, Liaoning Province, P R China

\section{Funding}

This work was supported by NSFC joint fund for regional innovation and development (No. U20A20413), National Natural Science Foundation of China (NSFC, No. 81972794, 81902708), Key R\&D Guidance Plan Projects in Liaoning Province (2019JH8/10300011), Shenyang S\&T Projects (20-204-4-22), Liaoning provincial department of education scientific research project (QN2019034) and Science and Technology Program of Liaoning Province (2017225036). Program for Shenyang High Level Talent Innovation and Entrepreneurship Team (2019-SYRCCY-B-01).

\section{Authors' contributions}

QLC,XML,XLL and MH conceived and designed the study. SQZ, XML collected and processed data. QLC,XML,SQZ and YYY analysed data.

QLC,XML,SQZ and JZ prepared tables and figures. QLC and XML drafted the manuscript. XLL and MH revised the manuscript. All authors read and approved the final manuscript.

\section{Acknowledgements}

Not applicable. 


\section{Web links and URLs:}

TCGA of the National Cancer Institute (TCGA, https://cancergenome.nih.gov/),STRING (version 11.0, https://string-db.org/),Metascape (http://metascape.org/),Oncomine (http://www.oncomine.org), GEPIA (http://gepia.cancer-pku.cn/),KM-plotter (http://www.kmplot.com/), TIMER (https://cistrome.shinyapps.io/timer/).

\section{References}

1. Siegel RL, Miller KD, Jemal A: Cancer statistics, 2020. CA Cancer J Clin 2020, 70(1):7-30.

2. de Martel C, Georges D, Bray F, Ferlay J, Clifford GM: Global burden of cancer attributable to infections in 2018: a worldwide incidence analysis. Lancet Glob Health 2020, 8(2):e180-e190.

3. Alfarouk KO, Bashir AHH, Aljarbou AN, Ramadan AM, Muddathir AK, AlHoufie STS, Hifny A, Elhassan GO, Ibrahim ME, Alqahtani SS et al: The Possible Role of Helicobacter pylori in Gastric Cancer and Its Management. Front Oncol 2019, 9:75.

4. Hooi JKY, Lai WY, Ng WK, Suen MMY, Underwood FE, Tanyingoh D, Malfertheiner P, Graham DY, Wong VWS, Wu JCY et al: Global Prevalence of Helicobacter pylori Infection: Systematic Review and Meta-Analysis. Gastroenterology 2017, 153(2):420-429.

5. Okada K, Suzuki S, Naito S, Yamada Y, Haruki S, Kubota M, Nakajima Y, Shimizu T, Ando K, Uchida Y et al: Incidence of metachronous gastric cancer in patients whose primary gastric neoplasms were discovered after Helicobacter pylori eradication. Gastrointest Endosc 2019, 89(6):1152-1159 e1151.

6. Venerito M, Vasapolli R, Rokkas T, Malfertheiner P: Gastric cancer: epidemiology, prevention, and therapy. Helicobacter 2018,23 Suppl 1:e12518.

7. Siegel RL, Miller KD, Fedewa SA, Ahnen DJ, Meester RGS, Barzi A, Jemal A: Colorectal cancer statistics, 2017. CA Cancer J Clin 2017, 67(3):177-193.

8. Siegel RL, Miller KD, Jemal A: Cancer Statistics, 2017. CA Cancer J Clin 2017, 67(1):7-30.

9. Friedmann-Morvinski D, Verma IM: Dedifferentiation and reprogramming: origins of cancer stem cells. EMBO Rep 2014, 15(3):244-253.

10. Bagheri V, Memar B, Behzadi R, Aliakbarian M, Jangjoo A, Bahar MM, Talebi S, Gholamin M, Abbaszadegan MR: Isolation and identification of chemotherapy-enriched sphere-forming cells from a patient with gastric cancer. J Cell Physio/ 2018, 233(10):7036-7046.

11. Chi HC, Tsai CY, Wang CS, Yang HY, Lo CH, Wang WJ, Lee KF, Lai LY, Hong JH, Chang YF et al: DOCK6 promotes chemo- and radioresistance of gastric cancer by modulating WNT/beta-catenin signaling and cancer stem cell traits. Oncogene 2020, 39(37):5933-5949.

12. Malta TM, Sokolov A, Gentles AJ, Burzykowski T, Poisson L, Weinstein JN, Kaminska B, Huelsken J, Omberg L, Gevaert 0 et al: Machine Learning Identifies Stemness Features Associated with Oncogenic Dedifferentiation. Cell 2018, 173(2):338-354 e315.

13. Pei G, Chen L, Zhang W: WGCNA Application to Proteomic and Metabolomic Data Analysis. Methods Enzymol 2017, 585:135-158.

14. Pei J, Wang Y, Li Y: Identification of key genes controlling breast cancer stem cell characteristics via stemness indices analysis. $J$ Trans/ Med 2020 , 18(1):74.

15. Bai KH, He SY, Shu LL, Wang WD, Lin SY, Zhang QY, Li L, Cheng L, Dai YJ: Identification of cancer stem cell characteristics in liver hepatocellular carcinoma by WGCNA analysis of transcriptome stemness index. Cancer Med 2020, 9(12):4290-4298.

16. Zhang Y, Tseng JT, Lien IC, Li F, Wu W, Li H: mRNAsi Index: Machine Leaming in Mining Lung Adenocarcinoma Stem Cell Biomarkers. Genes (Basel) 2020, 11(3)

17. Pan S, Zhan Y, Chen X, Wu B, Liu B: Identification of Biomarkers for Controlling Cancer Stem Cell Characteristics in Bladder Cancer by Network Analysis of Transcriptome Data Stemness Indices. Front Oncol 2019, 9:613.

18. Lvu W, Fei X, Chen C, Zhang B: In silico identification of the prognostic biomarkers and therapeutic targets associated with cancer stem cell characteristics of glioma. Biosci Rep 2020, 40(8).

19. Langfelder P, Horvath S: WGCNA: an R package for weighted correlation network analysis. BMC Bioinformatics 2008, 9:559.

20. Qu X, Tang Y, Hua S: Immunological Approaches Towards Cancer and Inflammation: A Cross Talk. Front Immuno/ $2018,9: 563$.

21. Vendramini-Costa DB, Carvalho JE: Molecular link mechanisms between inflammation and cancer. Curr Pharm Des 2012, 18(26):3831-3852.

22. Chung HW, Lim JB: Role of the tumor microenvironment in the pathogenesis of gastric carcinoma. World J Gastroentero/ 2014, 20(7):1667-1680.

23. Garcia-Gomez A, Rodriguez-Ubreva J, Ballestar E: Epigenetic interplay between immune, stromal and cancer cells in the tumor microenvironment. Clin Immunol 2018, 196:64-71.

24. Darre T, Kpatcha TM, Bagny A, Maneh N, Gnandi-Piou F, Tchangai B, Dare S, Adani-Ife S, Walla A, Amegbor K et al: Descriptive Epidemiology of Cancers in Togo from 2009 to 2016. Asian Pac J Cancer Prev 2017, 18(12):3407-3411.

25. Schistosomes, liver flukes and Helicobacter pylori. IARC Working Group on the Evaluation of Carcinogenic Risks to Humans. Lyon, 7-14 June 1994. IARC Monogr Eval Carcinog Risks Hum 1994, 61:1-241.

26. Sun DP, Lin CC, Hung ST, Kuang YY, Hseu YC, Fang CL, Lin KY: Aberrant Expression of NCAPG is Associated with Prognosis and Progression of Gastric Cancer. Cancer Manag Res 2020, 12:7837-7846.

27. Jin Y, Zhou K, Zhao W, Han R, Huo X, Yang F, Chen J: Clinicopathological and prognostic significance of metastasis-associated in colon cancer-1 in gastric cancer: A meta-analysis. Int J Biol Markers 2019, 34(1):27-32.

28. Ge S, Xia X, Ding C, Zhen B, Zhou Q, Feng J, Yuan J, Chen R, Li Y, Ge Z et al: A proteomic landscape of diffuse-type gastric cancer. Nat Commun 2018, 9(1):1012.

29. Eun K, Ham SW, Kim H: Cancer stem cell heterogeneity: origin and new perspectives on CSC targeting. BMB Rep 2017, 50(3):117-125.

30. Najafi M, Farhood B, Mortezaee K: Cancer stem cells (CSCs) in cancer progression and therapy. J Cell Physio/ 2019, 234(6):8381-8395.

Page 9/12 
31. Tong M, Yu C, Shi J, Huang W, Ge S, Liu M, Song L, Zhan D, Xia X, Liu W et al: Phosphoproteomics Enables Molecular Subtyping and Nomination of Kinase Candidates for Individual Patients of Diffuse-Type Gastric Cancer. iScience 2019, 22:44-57.

32. Bessede E, Dubus P, Megraud F, Varon C: Helicobacter pylori infection and stem cells at the origin of gastric cancer. Oncogene 2015, 34(20):2547-2555.

33. Tsugawa H, Kato C, Mori H, Matsuzaki J, Kameyama K, Saya H, Hatakeyama M, Suematsu M, Suzuki H: Cancer Stem-Cell Marker CD44v9-Positive Cells Arise From Helicobacter pylori-Infected CAPZA1-Overexpressing Cells. Cell Mol Gastroenterol Hepatol 2019, 8(3):319-334.

34. Yong X, Tang B, Xiao YF, Xie R, Qin Y, Luo G, Hu CJ, Dong H, Yang SM: Helicobacter pylori upregulates Nanog and Oct4 via Wnt/beta-catenin signaling pathway to promote cancer stem cell-like properties in human gastric cancer. Cancer Lett 2016, 374(2):292-303.

35. Browning RL, Mo X, Muthusamy N, Byrd JC: CpG oligodeoxynucleotide CpG-685 upregulates functional interleukin-21 receptor on chronic lymphocytic leukemia B cells through an NF-kappaB mediated pathway. Oncotarget 2015, 6(18):15931-15939.

36. Wood B, Sikdar S, Choi SJ, Virk S, Alhejaily A, Baetz T, LeBrun DP: Abundant expression of interleukin-21 receptor in follicular lymphoma cells is associated with more aggressive disease. Leuk Lymphoma 2013, 54(6):1212-1220.

37. Yan L, Zhang J, Guo D, Ma J, Shui SF, Han XW: IL-21R functions as an oncogenic factor and is regulated by the IncRNA MALAT1/miR-125a-3p axis in gastric cancer. Int $J$ Oncol 2019, 54(1):7-16.

38. Huang Y, Zhang J, Wang G, Chen X, Zhang R, Liu H, Zhu J: Oxymatrine exhibits anti-tumor activity in gastric cancer through inhibition of IL-21R-mediated JAK2/STAT3 pathway. Int J Immunopathol Pharmacol 2018, 32:2058738418781634.

39. Jia Z, Zhang Z, Yang Q, Deng C, Li D, Ren L: Effect of IL2RA and IL2RB gene polymorphisms on lung cancer risk. Int Immunopharmaco/ 2019 , 74:105716.

40. Mei L, Huang C, Wang A, Zhang X: Association between ADRB2, IL33, and IL2RB gene polymorphisms and lung cancer risk in a Chinese Han population. Int Immunopharmacol 2019, 77:105930.

41. Sugasawa $H$, Ichikura T, Tsujimoto $H$, Kinoshita M, Morita D, Ono S, Chochi K, Tsuda H, Seki S, Mochizuki H: Prognostic significance of expression of CCL5/RANTES receptors in patients with gastric cancer. J Surg Oncol 2008, 97(5):445-450.

42. Jeong EG, Kim MS, Nam HK, Min CK, Lee S, Chung YJ, Yoo NJ, Lee SH: Somatic mutations of JAK1 and JAK3 in acute leukemias and solid cancers. Clin Cancer Res 2008, 14(12):3716-3721.

43. Li SD, Ma M, Li H, Waluszko A, Sidorenko T, Schadt EE, Zhang DY, Chen R, Ye F: Cancer gene profiling in non-small cell lung cancers reveals activating mutations in JAK2 and JAK3 with therapeutic implications. Genome Med 2017, 9(1):89.

44. Inamdar VV, Reddy H, Dangelmaier C, Kostyak JC, Kunapuli SP: The protein tyrosine phosphatase PTPN7 is a negative regulator of ERK activation and thromboxane generation in platelets. J Biol Chem 2019, 294(33):12547-12554.

45. Murata M: Inflammation and cancer. Environ Health Prev Med 2018, 23(1):50.

46. Markopoulos GS, Roupakia E, Marcu KB, Kolettas E: Epigenetic Regulation of Inflammatory Cytokine-Induced Epithelial-To-Mesenchymal Cell Transition and Cancer Stem Cell Generation. Cells 2019, 8(10).

47. Chen Y, Zhang S, Wang Q, Zhang X: Tumor-recruited M2 macrophages promote gastric and breast cancer metastasis via M2 macrophage-secreted CHI3L1 protein. J Hematol Oncol 2017, 10(1):36.

48. Sica A, Mantovani A: Macrophage plasticity and polarization: in vivo veritas. J Clin Invest 2012, 122(3):787-795.

49. Newman AM, Liu CL, Green MR, Gentles AJ, Feng W, Xu Y, Hoang CD, Diehn M, Alizadeh AA: Robust enumeration of cell subsets from tissue expression profiles. Nat Methods 2015, 12(5):453-457.

50. Ritchie ME, Phipson B, Wu D, Hu Y, Law CW, Shi W, Smyth GK: limma powers differential expression analyses for RNA-sequencing and microarray studies. Nucleic Acids Res 2015, 43(7):e47.

51. Szklarczyk D, Morris JH, Cook H, Kuhn M, Wyder S, Simonovic M, Santos A, Doncheva NT, Roth A, Bork P et al: The STRING database in 2017: qualitycontrolled protein-protein association networks, made broadly accessible. Nucleic Acids Res 2017, 45(D1):D362-D368.

52. Rhodes DR, Yu J, Shanker K, Deshpande N, Varambally R, Ghosh D, Barrette T, Pandey A, Chinnaiyan AM: ONCOMINE: a cancer microarray database and integrated data-mining platform. Neoplasia 2004, 6(1):1-6.

53. Tang Z, Li C, Kang B, Gao G, Li C, Zhang Z: GEPIA: a web server for cancer and normal gene expression profiling and interactive analyses. Nucleic Acids Res 2017, 45(W1):W98-W102.

54. Li T, Fan J, Wang B, Traugh N, Chen Q, Liu JS, Li B, Liu XS: TIMER: A Web Server for Comprehensive Analysis of Tumor-Infiltrating Immune Cells. Cancer Res 2017, 77(21):e108-e110.

\section{Figures}




\section{Image not available with this version}

\section{Figure 1}

Correlation between corrected mRNAsi and clinical characteristics in H. pylori GC. (A) Differences in corrected mRNAsi between normal samples and samples of H. pylori GC. (B)The comparison of corrected mRNAsi of normal samples, H. pylori (-) GC samples and H. pylori (+) GC samples. (C) Kaplan-Meier survival curves of corrected mRNAsi in H. pylori GC.

\section{Image not available with this version}

Figure 2

Identification of DEGs and stemness-related key modules in GC. (A) DEGs: red indicates upregulated genes; blue indicates downregulated genes and black indicates genes excluded by DEG screening criteria. (B) Identification of a co-expression module in GC. Each piece of the leaves on the cluster dendrogram corresponded to a gene, and those genes with similar expression patterns compose a branch. (C) Correlation between gene modules and mRNAsi scores or EREG-mRNAsi. The first number of each module is the $\mathrm{R}$ value. The numbers in parentheses were $\mathrm{p}$-values, and $\mathrm{P}<0.05$ is considered statistically significant. (D, E) The scatter plot of the important gene modules in purple module and blue module. Each circle indicates a gene, and those circles located in the upper right indicate the key genes in these modules.

\section{Image not available with this version}

Figure 3

Co-expression Network and Enrichment Analysis of Key Genes. (A) Correlation between key genes at the transcriptional levels. (B) The protein-protein mutual interactions of key genes. (C-D) GO, KEGG and Reactome pathway analysis of key genes. (E) Detailed net structure of key genes through Metascape.

\section{Image not available with this version}


Expression validation of key genes. (A) The mRNA expression of key genes in GC samples and normal samples through GEPIA database analysis. (B) The mRNA expression of key genes in multiple cancer types from the Oncomine database. The number in the cells represents the number of analyses meeting the thresholds. Red indicates a higher expression level of target genes in tumor tissues than in normal tissues, and blue indicates an opposite expression pattern. The color depth of each cell indicates the gene rank, and the deeper the color depth is, the higher the gene rank.

\section{Image not available with this version}

Figure 5

The relationship between the expression levels of key genes related to stemness and the prognosis of GC patients. Kaplan-Meier survival plots (K-M plot) were generated using the online tool, Kaplan-Meier plotter. The log-rank test was used, and a $\mathrm{P}<0.001$ was considered as a significant difference. $\mathrm{K}-\mathrm{M}$ plots were drawn to profile a gene's prognostic value in GC, by the comparison of OS between the set of high-expression (Red curve) and the low (Black curve).

\section{Image not available with this version}

Figure 6

Correlation of mRNAsi key genes expression with immune infiltration level in GC.

\section{Supplementary Files}

This is a list of supplementary files associated with this preprint. Click to download.

- SupplementaryFigureS1.WGCNAresults.tif

- SupplementaryFigureS2.tif

- SupplementaryFigureS3.tif

- SupplementaryTables1.xlsx

- SupplementaryTables2.xlsx 